\title{
Sample Preparation and Analysis of Aggregated 'Single Atom Alloy' Nanoparticles by Atom Probe Tomography
}

\author{
Cédric Barroo ${ }^{1,2,3}$, Austin J. Akey ${ }^{4}$, Junjun Shan ${ }^{5}$, Maria Flytzani-Stephanopoulos ${ }^{5}$ and David C. Bell ${ }^{2,4}$ \\ 1. Chemical Physics of Materials and Catalysis, Université libre de Bruxelles, 1050 Brussels, Belgium \\ 2. John A. Paulson School of Engineering and Applied Sciences, Harvard University, Cambridge MA, \\ USA \\ 3. Department of Chemistry and Chemical Biology, Harvard University, Cambridge MA, USA \\ 4. Center for Nanoscale Systems, Harvard University, Cambridge MA, USA \\ 5. Department of Chemical and Biological Engineering, Tufts University, Medford MA, USA
}

Atom probe tomography (APT) is a powerful technique for the characterization of composition of materials and their three-dimensional structure down to the atomic-scale. With the new developments in FIB-based sample preparation, there is a growing interest in the analysis of catalytic systems by APT [1]. Our current research focuses on the use of nanostructured materials for sustainable catalysis applications, and more specifically, on metallic alloy catalyst materials for selective oxidation or dehydrogenation reaction. The principal design feature of the catalyst material is to combine a minor amount of active metal that facilitates creation of reactive intermediates with a less active majority phase that transforms these intermediates to desirable products with high selectivity.

Copper-based catalysts, in the form of single atom alloy (SAA) catalysts, are currently being developed for selective dehydrogenation reactions using a modified electroless galvanic deposition method [2]. $\mathrm{CuNi}_{0.01}$ nanoparticles were synthesized on graphite to avoid the presence of a porous support which may affect the 3D reconstruction. The incorporation of atomically dispersed $\mathrm{Ni}$ within $\mathrm{Cu}$ catalysts (as both nanoparticles and nanoporous structure) allows lowering the activation energy for dehydrogenation of alcohols. The typical concentration of $\mathrm{Ni}$ on these supported $\mathrm{CuNi}$ nanoparticles is 0.01 or $1 \%$ atomic, and it is difficult to localise the $\mathrm{Ni}$ atoms with regular microscopy techniques. APT should be particularly suited to determine the distribution of $\mathrm{Ni}$ within the nanoparticles.

The different pathways for the preparation of atom probe samples from nanoparticles consist mainly in dielectrophoresis, drying/incubation, and agglomerate lift-out (in the case of powder samples) [3]. However, after reduction in $\mathrm{H}_{2}$, the catalyst appears in the form of isolated 12-15 nm nanoparticles and agglomeration of nanoparticles into 1-3 $\mu \mathrm{m}$ clusters (see Figure 1(left)). It has to be noted that the dispersion of nanoparticles is too low to use the drying/incubation techniques. To prepare samples suitable for APT analysis, clustered nanoparticles were attached to an Omniprobe tip with Pt-weld in the FIB, and the agglomerate was then sharpened down to a radius of $\approx 50 \mathrm{~nm}$. The agglomeration of NPs induces the formation of a porous structure, which has been filed with Pt-weld at every step of the sample preparation (before and after annular milling) to avoid as much as possible the presence of aberration and sample fracture due to the presence of porosity.

Fresh catalysts (before any reaction) were analysed by APT. Experiments were performed in a LEAP 4000X HR system with conditions of acquisition: $40 \mathrm{pJ}, 100 \mathrm{kHz}, 50 \mathrm{~K}, \mathrm{DR}$ : $0.25 \%$. Even though the structure has been filled as much as possible, the presence of voids in the agglomerate is inevitable, and induces micro-fractures which are reflected in the voltage plot. As it can be seen on Figure 1(right), the $3 \mathrm{D}$ reconstruction displays the presence of $\approx 15 \mathrm{~nm}$ particles. The mass spectrum indicates the presence 
of $\mathrm{Cu}, \mathrm{CuH}$ and $\mathrm{Cu}_{\mathrm{x}} \mathrm{O}_{\mathrm{y}}$ species. The copper hydrates are due to the initial pre-treatment, and the copper oxides are believed to be due to the exposure of the sample to air after FIB. To determine the presence of $\mathrm{Ni}$ and/or the presence of clustering in this sample, further data treatment has been performed, as well as comparative STEM-EELS measurements.

In conclusion, this study has proved that, even though the structure is not optimal, it is possible to prepare APT samples from agglomeration of nanoparticles using a rather fast technique, which does not require deposition and incubation of the nanoparticles. This procedure could also be used in future developments towards the analysis of porous materials by atom probe tomography. Future research will focus on the study of SAA catalysts on different supports, as well as the evolution of the composition at different steps of the catalytic reaction [4].

\section{References:}

[1] C. Barroo et al, Atomically-Precise Methods for Synthesis of Solid Catalysts (2015), p. 248.

[2] J. Shan et al, Appl. Catal. B: Environ. 205 (2017), p. 541.

[3] P. Felfer et al, Ultramicroscopy 159 (2015), p. 413.

[4] This work was supported as part of the Integrated Mesoscale Architectures for Sustainable Catalysis - IMASC, an Energy Frontier Research Center funded by the U.S. Department of Energy, Office of Science, Basic Energy Sciences under Award \# DE-SC0012573. C.B. acknowledges postdoctoral fellowships through the Belgian American Educational Foundation (BAEF) as well as WallonieBruxelles International (Excellence grant WBI.WORLD) foundations.
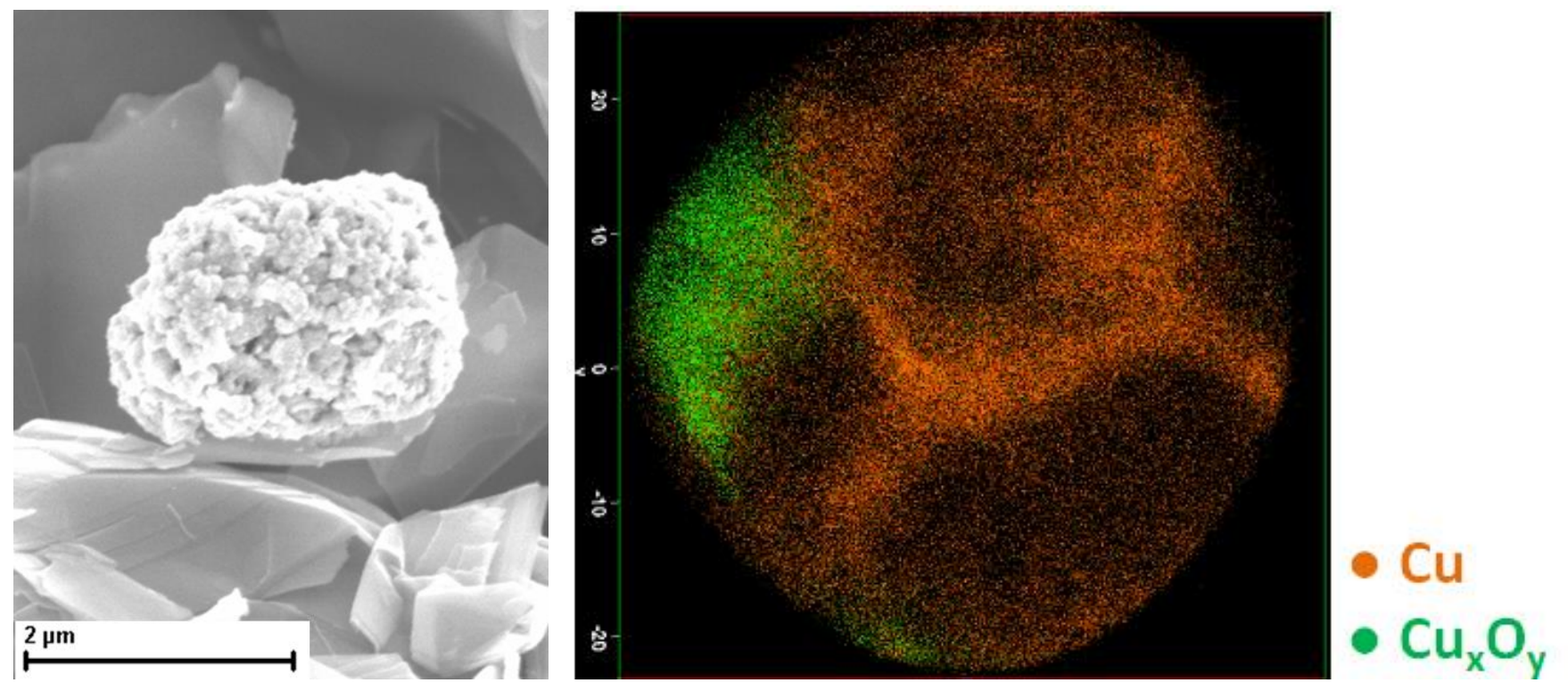

Figure 1. (left) SEM image of the agglomeration of SAA catalyst nanoparticles supported on graphite (right) APT reconstruction: 2D projection of a selected region of the sample allowing discerning the presence of nanoparticles, and showing the partial oxidation of the sample (scale is in $\mathrm{nm}$ ) 